\title{
Complexity and heights of tori
}

\author{
Gautam Chinta Jay Jorgenson Anders Karlsson *
}

October 31, 2018

\begin{abstract}
We prove detailed asymptotics for the number of spanning trees, called complexity, for a general class of discrete tori as the parameters tend to infinity. The proof uses in particular certain ideas and techniques from an earlier paper CJK10. Our asymptotic formula provides a link between the complexity of these graphs and the height of associated real tori, and allows us to deduce some corollaries on the complexity thanks to certain results from analytic number theory. In this way we obtain a conjectural relationship between complexity and regular sphere packings.
\end{abstract}

\section{Introduction}

The number of spanning trees $\tau(G)$, called the complexity, of a finite graph $G$ is an invariant which is of interest in several sciences: network theory, statistical physics, theoretical chemistry, etc. Via the well-known matrix-tree theorem of Kirchoff, the complexity equals the determinant of the combinatorial Laplacian $\Delta_{G}$ divided by the number of vertices.

For compact Riemannian manifolds $M$ there is an analogous invariant $h(M)$, the height, defined as the negative of the logarithm of the zetaregularized determinant of the Laplace-Beltrami operator, and which is of interest for quantum physics. The analogy between the height and complexity has been commented on by Sarnak in [S90].

In statistical physics it is of interest to study the asymptotics of the complexity, and other spectral invariants, for certain families of graphs. Important cases to study are various subgraphs of the standard lattice $\mathbb{Z}^{d}$. An

${ }^{*}$ The first and second authors acknowledge support provided by grants from the National Science Foundation and the Professional Staff Congress of the City University of New York. The third author received support from SNSF grant 200021_132528/1. 
instance of this is to study discrete tori, corresponding to periodic boundary conditions, as the parameters tend to infinity, see [DD88, [CJK10], and references therein. It is shown in [CJK10] that in the asymptotics of the complexity of discrete tori, the height of an associated real torus appears as a constant.

In the present paper we study discrete tori of a more general type, defined as follows. Let $\Lambda$ be an invertible $r \times r$ matrix with all entries being integers. This matrix defines a lattice $\Lambda \mathbb{Z}^{r}$ in $\mathbb{R}^{r}$. We associate to the group quotient with standard generators

$$
\Lambda \mathbb{Z}^{r} \backslash \mathbb{Z}^{r}
$$

its Cayley graph, which we call a discrete torus. In other words, two elements $x$ and $y$ in $\Lambda \mathbb{Z}^{r} \backslash \mathbb{Z}^{r}$ are adjacent, denoted $x \sim y$, if they differ by \pm 1 in exactly one of the coordinates and equal everywhere else (everything mod $\Lambda \mathbb{Z}^{r}$ of course).

Let $0=\lambda_{0}<\lambda_{1} \leq \ldots \leq \lambda_{|\operatorname{det} \Lambda|-1}$ be the eigenvalues of the combinatorial Laplacian $\Delta_{\Lambda \mathbb{Z}^{r} \backslash \mathbb{Z}^{r}}$ of the discrete torus - see (1) for a definition of $\Delta_{\Lambda \mathbb{Z}^{r} \backslash \mathbb{Z}^{r}}$. Define $\operatorname{det}^{*} \Delta_{\Lambda \mathbb{Z}^{r} \backslash \mathbb{Z}^{r}}$ to be the product of the nonzero eigenvalues of the Laplacian:

$$
\operatorname{det}^{*} \Delta_{\Lambda \mathbb{Z}^{r} \backslash \mathbb{Z}^{r}}:=\lambda_{1} \lambda_{2} \ldots \lambda_{|\operatorname{det} \Lambda|-1} .
$$

Note that the trivial eigenvalue is removed. We will nevertheless refer to $\operatorname{det}^{*} \Delta_{\Lambda \mathbb{Z}^{r} \backslash \mathbb{Z}^{r}}$ as the determinant of the Laplacian. For simplicity we will mostly assume that $\operatorname{det} \Lambda>0$.

Theorem 1 Let $\left\{\Lambda_{n}\right\}$ be a sequence of $r \times r$ integer matrices. Suppose that $\operatorname{det} \Lambda_{n} \rightarrow \infty$ and $\Lambda_{n} /\left(\operatorname{det} \Lambda_{n}\right)^{1 / r} \rightarrow A \in S L_{r}(\mathbb{R})$. Then as $n \rightarrow \infty$,

$$
\log \operatorname{det}^{*} \Delta_{\Lambda_{n} \mathbb{Z}^{r} \backslash \mathbb{Z}^{r}}=c_{r} \operatorname{det} \Lambda_{n}+\frac{2}{r} \log \operatorname{det} \Lambda_{n}+\log \operatorname{det}^{*} \Delta_{A \mathbb{Z}^{r} \backslash \mathbb{R}^{r}}+o(1)
$$

where

$$
c_{r}=\log 2 r-\int_{0}^{\infty} e^{-2 r t}\left(I_{0}(2 t)^{r}-1\right) \frac{d t}{t} .
$$

The definition of $\log \operatorname{det}^{*} \Delta_{A \mathbb{Z}^{r} \backslash \mathbb{R}^{r}}$ will be recalled in section 3 below.

Our earlier paper [CJK10] treats the case when the $\Lambda_{n}$ are diagonal matrices. The present paper uses several facts that are established in that paper. Thanks to the fact that the first two terms in the asymptotics are universal in the sense that they only depend on $\Lambda_{n}$ via $\operatorname{det} \Lambda_{n}$, the theorem gives a close connection between the complexity of certain graphs and the height of an associated manifold. We emphasize that this is not at all obvious: 
while it is true that the appropriately rescaled eigenvalues of the discrete tori converge to the eigenvalues of $\Delta_{A \mathbb{Z}^{r} \backslash \mathbb{R}^{r}}$, this convergence is certainly not uniform. Moreover, the height cannot be defined as the product of eigenvalues, there is a regularization in the definition. An explicit expression for the height of flat tori of volume $1, h\left(A \mathbb{Z}^{r} \backslash \mathbb{R}^{r}\right):=-\log \operatorname{det} \Delta_{A \mathbb{Z}^{r} \backslash \mathbb{R}^{r}}$, can be found in Theorem 2.3 of [Ch97]. Deninger and Lück have informed us that the constant $c_{r}$ also has an interpretation as a determinant, namely the Fuglede-Kadison determinant of the Laplacian on $\mathbb{Z}^{r}$, see $[\mathrm{L}]$.

We turn now to a connection between our results and sphere packings. The problem of finding the densest packing of ordinary space with spheres of equal radii is an old one with practical importance even in dimensions greater than 3. One type of packings is regular sphere packings which means that the spheres are centered at the points of a lattice $A \mathbb{Z}^{r}$. Gauss showed that the face centered lattice (fcc) $D_{3}$ is optimal among regular packings in three dimensions. By work of Thue and Toth one knows that the hexagonal lattice $A_{2}$ is densest in dimension 2. In dimenison 24 it is known that the Leech lattice is optimal among unimodular lattices. We refer to the book of Conway-Sloane CS99 for more information.

Conjecturally the height of $A \mathbb{Z}^{r} \backslash \mathbb{R}^{r}$ has a global minimum when $A \mathbb{Z}^{r}$ is the densest regular sphere packing. Extremal metrics for heights has been studied in OPS88 in dimension 2, and for tori in higher dimensions notably in Ch97] and [SS06]. In these papers, the question is phrased as the study of the derivative of Epstein zeta functions at $s=0$. From this theory we can deduce the following corollaries from our main theorem:

Corollary 2 Let $\left\{\Lambda_{n}\right\}$ be a sequence of $r \times r$ integer matrices with $\operatorname{det} \Lambda_{n} \rightarrow$ $\infty$. Suppose that $\left\{\Lambda_{n} /\left(\operatorname{det} \Lambda_{n}\right)^{1 / r}\right\}$ belongs to a compact subset of $S L_{r}(\mathbb{R})$, $r=2,3$, avoiding lattices equivalent to $A_{2}$, resp. $D_{3}$. Assume that there is a sequence $\left\{L_{n}\right\}$ with $\operatorname{det} L_{n}=\operatorname{det} \Lambda_{n}$ such that $\left\{L_{n} /\left(\operatorname{det} L_{n}\right)^{1 / r}\right\}$ converges to $A_{2}$, resp. $D_{3}$. Then $L_{n} \mathbb{Z}^{r} \backslash \mathbb{Z}^{r}$ has more spanning trees than $\Lambda_{n} \mathbb{Z}^{r} \backslash \mathbb{Z}^{r}$ for all sufficiently large $n$.

Corollary 3 Let $\Lambda_{n}$ be a sequence of $r \times r$ integer matrices with $\operatorname{det} \Lambda_{n} \rightarrow \infty$ Suppose that $\left\{\Lambda_{n} /\left(\operatorname{det} \Lambda_{n}\right)^{1 / r}\right\}$ stays in a compact subset of $S L_{r}(\mathbb{R})$. For all sufficiently large $n$ we have that

$$
\tau\left(\Lambda_{n} \mathbb{Z}^{r} \backslash \mathbb{Z}^{r}\right) \leq \frac{\left(\operatorname{det} \Lambda_{n}\right)^{2 / r-1}}{4 \pi} \exp \left(c_{r} \operatorname{det} \Lambda_{n}+\gamma+2 / r\right),
$$

where $\gamma$ is Euler's constant and $c_{r}$ is as in the theorem. 
In the trivial case $r=1$, this estimate gives a value close to the truth:

$$
\operatorname{det} \Lambda_{n}=\tau\left(\Lambda_{n} \mathbb{Z}^{r} \backslash \mathbb{Z}^{r}\right) \leq 1.05 \operatorname{det} \Lambda_{n} .
$$

Upper bounds for the number of spanning trees have been considered in the combinatorics literature since 1970s at least. For regular graphs there is a rather sharp estimate by Chung and Yau CY99 improving on an earlier result of McKay [M]. In general, it is an open problem to decide which simple graph on $n$ vertices and $e$ edges has the maximal complexity. This is of interest to communication network theory since this graph invariant appears as a measure of reliability.

It would be of interest to also go in the other direction: proving results on the extrema of families of Epstein zeta functions via a better understanding of the number of spanning trees of discrete tori.

\section{Spectral preliminaries for discrete tori}

Let $\Lambda$ be an invertible $r \times r$ integer matrix. This matrix defines a lattice $\Lambda \mathbb{Z}^{r}$ in $\mathbb{R}^{r}$. We denote by $D T(\Lambda)$ the discrete torus, or Cayley graph of the quotient group $\Lambda \mathbb{Z}^{r} \backslash \mathbb{Z}^{r}$ with standard generating set: two elements $x$ and $y$ in $\Lambda \mathbb{Z}^{r} \backslash \mathbb{Z}^{r}$ are adjacent, denoted $x \sim y$, if they differ by \pm 1 in exactly one of the coordinates and equal everywhere else (everything $\bmod \Lambda \mathbb{Z}^{r}$ of course).

The associated (combinatorial) Laplacian is defined by

$$
\Delta_{D T(\Lambda)} f(x)=\sum_{y \text { s.t. } y \sim x}(f(x)-f(y))
$$

on functions $f: \Lambda \mathbb{Z}^{r} \backslash \mathbb{Z}^{r} \rightarrow \mathbb{R}$.

The dual lattice $\Lambda^{*} \mathbb{Z}^{r}$ is as usual all the points $v$ in $\mathbb{R}^{r}$ such that $(v, x) \in \mathbb{Z}$ for all $x \in \Lambda \mathbb{Z}^{r}$, where $(\cdot, \cdot)$ denotes the usual scalar product. Since $\mathbb{Z}^{r}$ is self-dual and $\Lambda \mathbb{Z}^{r}$ is a subgroup of $\mathbb{Z}^{r}$ it follows that $\mathbb{Z}^{r}$ is a subgroup of $\Lambda^{*} \mathbb{Z}^{r}$. Note that the respective indices are

$$
\left[\mathbb{Z}^{r}: \Lambda \mathbb{Z}^{r}\right]=\left[\Lambda^{*}: \mathbb{Z}^{r}\right]=|\operatorname{det} \Lambda|
$$

Proposition 4 The eigenfunctions of $\Delta_{D T(\Lambda)}$ are given by

$$
f_{v}(x)=e^{2 \pi i(x, v)}
$$

for each $v \in \mathbb{Z}^{r} \backslash \Lambda^{*} \mathbb{Z}^{r}$, with corresponding eigenvalue given by

$$
\lambda_{v}=2 r-2 \sum_{k=1}^{r} \cos \left(2 \pi v_{k}\right),
$$


where $v_{k}$ denotes the $k$ th coordinate of $v$.

Proof. The operator $\Delta_{D T(\Lambda)}$ is a semipositive, symmetric matrix and hence we are looking for $|\operatorname{det} \Lambda|$ number of eigenfunctions and eigenvalues. The proof is a trivial calculation:

$$
\begin{aligned}
\Delta e^{2 \pi i(x, v)} & =2 r e^{2 \pi i(x, v)}-\sum_{y \sim x} e^{2 \pi i(y, v)}= \\
& =\left(2 r-\sum_{z \sim 0} e^{2 \pi i(z, v)}\right) e^{2 \pi i(x, v)} .
\end{aligned}
$$

The heat kernel $K^{\Lambda}(t, x): \mathbb{R}_{\geq 0} \times D T(\Lambda) \rightarrow \mathbb{R}$ is the unique bounded function which satisfies

$$
\begin{aligned}
\left(\Delta_{D T(\Lambda)}+\frac{\partial}{\partial t}\right) K^{\Lambda}(t, x) & =0 \\
K^{\Lambda}(0, x) & =\delta_{0}(x),
\end{aligned}
$$

where $\delta_{0}(x)=1$ if $x=0$ and 0 otherwise. The existence and uniqueness of heat kernels in a general graph setting is established in [DM06]. Recall from e.g. CJK10, that

$$
K^{\mathbb{Z}^{r}}(t, z)=\prod_{k=1}^{r} K^{\mathbb{Z}}\left(t, z_{k}\right)
$$

for $z=\left(z_{k}\right)$ and $K^{\mathbb{Z}}(t, w)=e^{-2 t} I_{w}(2 t)$, where $I_{w}$ is the $I$-Bessel function of order $w$.

We have the following theta inversion formula (cf. [CJK10]).

Proposition 5 The following formula holds for $x \in \Lambda \mathbb{Z}^{r} \backslash \mathbb{Z}^{r}$ and $t \in \mathbb{R}_{\geq 0}$

$$
\sum_{y \in \Lambda \mathbb{Z}^{r}} K^{\mathbb{Z}^{r}}(t, x-y)=\frac{1}{|\operatorname{det} \Lambda|} \sum_{\nu \in \mathbb{Z}^{r} \backslash \Lambda^{*} \mathbb{Z}^{r}} e^{-t \lambda_{v}} f_{v}(x) .
$$

In particular,

$$
\theta_{\Lambda}(t):=|\operatorname{det} \Lambda| \sum_{y \in \Lambda \mathbb{Z}^{r}} e^{-2 r t} I_{y_{1}}(2 t) \ldots I_{y_{r}}(2 t)=\sum_{\nu \in \mathbb{Z}^{r} \backslash \Lambda^{*} \mathbb{Z}^{r}} e^{-t \lambda_{v}}
$$

Proof. Since both sides of the equation satisfy the conditions for being the heat kernel, this follows from the uniqueness of heat kernels. The second formula is the special case $x=0$. 


\section{Spectral preliminaries for continuous tori}

An $r$-dimensional (continuous) torus is given as a quotient of $\mathbb{R}^{\mathrm{r}}$ by a lattice $A \mathbb{Z}^{\mathrm{r}}$ where $A \in G L_{r}(\mathbb{R})$. The metric structure and the standard (positive) Laplace-Beltrami operator $-\sum_{i} \partial^{2} / \partial x_{i}^{2}$ on $\mathbb{R}^{\mathrm{r}}$ projects to the torus. The volume is $|\operatorname{det} A|$. Let $A^{*}$ be the matrix defining the dual lattice $A^{*} \mathbb{Z}^{\mathrm{r}}$, and so $A^{*}=\left(A^{-1}\right)^{t}$. The eigenfunctions of the Laplacian on the torus in question are $f_{v}(x)=\exp \left(2 \pi i v^{t} x\right)$ where $v$ are the vectors in the dual lattice. The corresponding eigenvalues are $\lambda_{v}=4 \pi^{2}\|v\|^{2}$ or with a different indexing: $\lambda_{m}=(2 \pi)^{2}\left(A^{*} m\right)^{t}\left(A^{*} m\right)$, where $m$ runs through $\mathbb{Z}^{r}$. We have the associated theta function

$$
\Theta_{A}(t)=\sum_{m \in \mathbb{Z}^{r}} e^{-(2 \pi)^{2}\left(A^{*} m\right)^{t}\left(A^{*} m\right) \cdot t}
$$

The theta inversion formula, which is equivalent to the Poisson summation formula in this case, yields

$$
\Theta_{A}(t)=\frac{1}{(4 \pi t)^{r / 2}} \sum_{x \in A \mathbb{Z}^{r}} e^{-|x|^{2} / 4 t} .
$$

The associated spectral zeta function, which in this case also goes under the name of the Epstein zeta function, is defined as

$$
Z_{A}(s)=\sum_{m \neq 0} \lambda_{m}^{-s}=\frac{1}{(2 \pi)^{2 s}} \sum_{v \neq 0} \frac{1}{\|v\|^{2 s}} .
$$

Classically, one can prove the meromorphic continuation of $Z_{A}(s)$ to all $s \in \mathbb{C}$, showing that its continuation is holomorphic at $s=0$. From this, one defines the spectral determinant $\operatorname{det}^{*} \Delta_{A \mathbb{Z}^{r} \backslash \mathbb{R}^{r}}$ by

$$
\log \operatorname{det}^{*} \Delta_{A \mathbb{Z}^{r} \backslash \mathbb{R}^{r}}=-Z_{A}^{\prime}(0) .
$$

\section{Asymptotics}

Let

$$
\mathcal{I}_{r}(s)=-\int_{0}^{\infty}\left(e^{-s^{2} t} e^{-2 r t} I_{0}(2 t)^{r}-e^{-t}\right) \frac{d t}{t}
$$

and

$$
\mathcal{H}_{\Lambda}(s)=-\int_{0}^{\infty}\left(e^{-s^{2} t}\left[\theta_{\Lambda}(t)-|\operatorname{det} \Lambda| \cdot e^{-2 r t} I_{0}(2 t)^{r}-1\right]+e^{-t}\right) \frac{d t}{t} .
$$


Everything in section 3 of [CJK10] carries over with only notational changes, even though the eigenvalues are different and the theta identity is hence somewhat different. These differences are not essentially used. In particular the first order term as $t \rightarrow 0$ in the trace of the heat kernel is still (in the present notation) $|\operatorname{det} \Lambda| \cdot e^{-2 r t} I_{0}(2 t)^{r}$ since it corresponds to the trivial eigenvalue. In particular the following extension of Theorem 3.6 in [CJK10] holds:

Theorem 6 For any $s \in \mathbb{C}$ with $\operatorname{Re}\left(s^{2}\right)>0$, we have the relation

$$
\sum_{\lambda_{v} \neq 0} \log \left(s^{2}+\lambda_{\nu}\right)=|\operatorname{det} \Lambda| \cdot \mathcal{I}_{r}(s)+\mathcal{H}_{\Lambda}(s) .
$$

Letting $s \rightarrow 0$ we have the identity

$$
\log \left(\prod_{\lambda_{v} \neq 0} \lambda_{\nu}\right)=|\operatorname{det} \Lambda| \cdot \mathcal{I}_{r}(0)+\mathcal{H}_{\Lambda}(0) .
$$

Section 4 of CJK10 is an independent section on uniform bounds on $I$-Bessel functions. We recall the following statements, slightly adapted to the present context (keeping in mind that $I_{-y}=I_{y}$ for integers, and that $b$ may now be real):

Proposition 7 For any $t>0$ and $b \geq 0$, there is a constant $C$ such that

$$
0 \leq \sqrt{b^{2} t} e^{-b^{2} t} I_{0}\left(b^{2} t\right) \leq C<1
$$

Fix $t \geq 0$ and integers $y, n_{0} \geq 0$. Then for all $b \geq n_{0}$ we have the uniform bound

$$
0 \leq \sqrt{b^{2} t} \cdot e^{-b^{2} t} I_{y}\left(b^{2} t\right) \leq\left(1+\frac{y}{b n_{0} t}\right)^{-n_{0} y / 2 b} .
$$

Proposition 8 Let $N(u)$ be a sequence of positive integers parametrized by $u \in \mathbb{Z}_{+}$such that $N(u) / u \rightarrow \alpha>0$ as $u \rightarrow \infty$. Then for any $t>0$ and integer $k$, we have

$$
\lim _{u \rightarrow \infty} N(u) e^{-2 u^{2} t} I_{N(u) k}\left(2 u^{2} t\right)=\frac{\alpha}{\sqrt{4 \pi t}} e^{-(\alpha k)^{2} / 4 t} .
$$

From now on we fix a sequence $\left\{\Lambda_{n}\right\}$ of integer matrices with $0<$ $\operatorname{det} \Lambda_{n} \rightarrow \infty$ satisfying

$$
\frac{1}{\left(\operatorname{det} \Lambda_{n}\right)^{1 / r}} \Lambda_{n} \rightarrow A \text { as } n \rightarrow \infty
$$

for some $A \in \mathrm{SL}_{r}(\mathbb{R})$. From the previous propositions we will deduce the following: 
Proposition 9 For each fixed $t>0$ we have the pointwise convergence

$$
\theta_{\Lambda_{n}}\left(\operatorname{det}\left(\Lambda_{n}\right)^{2 / r} t\right) \rightarrow \theta_{A}(t)
$$

as $n \rightarrow \infty$.

Proof. For any $v \in \mathbb{Z}^{r}$ and $\Lambda \in \mathrm{GL}_{r}(\mathbb{R})$, let

$$
\mathbf{I}_{v, \Lambda}(t)=\prod_{i=1}^{r} I_{(\Lambda v)_{i}}(t)
$$

where $(\Lambda v)_{i}$ denotes the $i$-th component of $\Lambda v$. Let $u_{n}=\operatorname{det}\left(\Lambda_{n}\right)^{1 / r}$ and $a_{i}=(A v)_{i}$. Note that $(\Lambda v)_{i} / u_{n} \rightarrow a_{i}$. We have

$$
\theta_{\Lambda_{n}}\left(u_{n}^{2} t\right)=\sum_{v \in \mathbb{Z}^{r}} u_{n}^{r} e^{-2 r u_{n}^{2} t} \mathbf{I}_{v, \Lambda_{n}}\left(2 u_{n}^{2} t\right)
$$

From Proposition 8 (with $k=0$ or \pm 1 ) we have for any $t>0$ and $v \in \mathbb{Z}^{r}$ that

$$
u_{n}^{r} e^{-2 r u_{n}^{2} t} \mathbf{I}_{v, \Lambda_{n}}\left(2 u_{n}^{2} t\right) \rightarrow \frac{1}{(\sqrt{4 \pi t})^{r}} e^{-a_{1}^{2} / 4 t} \ldots e^{-a_{r}^{2} / 4 t}
$$

as $n \rightarrow \infty$. This means that the proposition will be proved if we can interchange the limit and the infinite sum. We show that for fixed $t$, the sum is convergent uniformly in $u_{n}$ (or equivalently, in $n$ ).

We can rewrite the sum $\theta_{\Lambda_{n}}\left(u_{n}^{2} t\right)$ in $r+1$ sums depending on how many components of the $\Lambda_{n} v$ are zero. Pick $n_{0}$ sufficiently large so that

$$
\left|a_{i}\right| / 2 \leq\left|\left(\Lambda_{n} v\right)_{i}\right| / u_{n} \leq 2\left|a_{i}\right|
$$

for all $v \in \mathbb{Z}^{r}$ and $n \geq n_{0}$ and $a_{i} \neq 0$. Recall that $I_{-n}=I_{n}$. Let us look at a term with $k$ zeros in the $y_{i}$ s and estimate with the help of Proposition 7 ;

$$
\begin{aligned}
u_{n}^{r} e^{-2 r u_{n}^{2} t} \mathbf{I}_{v, \Lambda_{n}}\left(2 u_{n}^{2} t\right) & \leq\left(\frac{1}{\sqrt{2 t}}\right)^{r} \prod_{\left(\Lambda_{n} v\right)_{i} \neq 0}\left(1+\frac{\left|\left(\Lambda_{n} v\right)_{i}\right|}{u_{n} n_{0} 2 t}\right)^{-n_{0}\left|\left(\Lambda_{n} v\right)_{i}\right| / 2 u_{n}} \\
& \leq\left(\frac{1}{\sqrt{2 t}}\right)^{r} \prod_{a_{i} \neq 0} \lambda^{\left|a_{i}\right|}
\end{aligned}
$$

for all $n$ large and where

$$
\lambda:=\left(1+\frac{a}{n_{0} 4 t}\right)^{-n_{0} / 4}<1
$$


and $a$ is the smallest nonzero absolute value of all the entries in $A \mathbb{Z}^{r}$. The whole theta series is therefore bounded by $r+1$ sums of a product of convergent geometric series. This shows that the infinite sum is uniformly convergent and the proof is complete.

Lemma 10 Given a sequence $\left\{\Lambda_{n}\right\}$ satisfying $\left(\operatorname{det} \Lambda_{n}\right)^{-1 / r} \Lambda_{n} \rightarrow A$ as above, there is a constant $d>0$ such that for all sufficiently large $n$

$$
\theta_{\Lambda_{n}}\left(u_{n}^{2} t\right) \leq 1+\sum_{j=1}^{\infty} e^{-d t j} .
$$

Proof. Let $u_{n}=\operatorname{det}\left(\Lambda_{n}\right)^{1 / r}$. We have

$$
\begin{aligned}
\theta_{\Lambda_{n}}(t) & =\sum_{\nu \in \mathbb{Z}^{r} \backslash \Lambda_{n}^{*} \mathbb{Z}^{r}} e^{-t \lambda_{v}}=\sum_{\nu \in \mathbb{Z}^{r} \backslash \Lambda_{n}^{*} \mathbb{Z}^{r}} e^{-t\left(2 r-2 \sum_{k=1}^{r} \cos \left(2 \pi v_{k}\right)\right)} \\
& =1+\sum_{\substack{\nu \in \mathbb{Z}^{r} \backslash \Lambda_{n}^{*} \mathbb{Z}^{r} \\
v \neq 0}} \prod_{k=1}^{r} e^{-4 t \sin ^{2}\left(\pi v_{k}\right)}
\end{aligned}
$$

So that

$$
\theta_{\Lambda_{n}}\left(u_{n}^{2} t\right)=1+\sum_{\substack{\nu \in \mathbb{Z}^{r} \backslash \Lambda_{n}^{*} \mathbb{Z}^{r} \\ v \neq 0}} \prod_{k=1}^{r} e^{-4 t u_{n}^{2} \sin ^{2}\left(\pi v_{k}\right)}
$$

We use the elementary bounds $\sin x \geq x-x^{3} / 6$ and $\sin (\pi-x) \geq x-x^{3} / 6$ for $x \in[0, \pi / 2]$ and get

$$
\begin{aligned}
& u_{n} \sin \left(\pi v_{k}\right) \geq u_{n} \pi v_{k}\left(1-\pi^{2} v_{k}^{2} / 6\right)>c \pi u_{n} v_{k} \text { if } v_{k} \leq 1 / 2 \\
& u_{n} \sin \left(\pi v_{k}\right) \geq u_{n} \pi v_{k}\left(1-\pi^{2} v_{k}^{2} / 6\right)>c \pi u_{n}\left(1-v_{k}\right) \text { if } v_{k}>1 / 2
\end{aligned}
$$

for some postitve constant $c$ for all $n$ sufficiently large. Note also that for every $v \neq 0$, the values $u_{n} v_{k}$ range over the integers times an entry in $A$ as $n \rightarrow \infty$ because of the convergence of $\left(\operatorname{det} \Lambda_{n}\right)^{-1 / r} \Lambda_{n}$ to $A$ in $\mathrm{SL}_{r}(\mathbb{R})$. We then conclude there is a constant $d>0$ such that for all sufficiently large $n$

$$
\theta_{\Lambda_{n}}\left(u_{n}^{2} t\right) \leq 1+\sum_{j=1}^{\infty} e^{-d t j} .
$$

Now we can show: 
Proposition 11 With the notation as above and $u_{n}:=\operatorname{det}\left(\Lambda_{n}\right)^{1 / r}$, we have that

$$
\begin{aligned}
& \int_{1}^{\infty}\left(\theta_{\Lambda_{n}}\left(u_{n}^{2} t\right)-u_{n}^{r} e^{-2 r u_{n}^{2} t} I_{0}\left(2 u_{n}^{2} t\right)^{r}-1+e^{-u_{n}^{2} t}\right) \frac{d t}{t} \\
& =\int_{1}^{\infty}\left(\theta_{A}(t)-1\right) \frac{d t}{t}-\frac{2}{r}(4 \pi)^{-r / 2}+o(1)
\end{aligned}
$$

as $n \rightarrow \infty$

Proof. Write

$$
\begin{aligned}
& \int_{1}^{\infty}\left(\theta_{\Lambda_{n}}\left(u_{n}^{2} t\right)-u_{n}^{r} e^{-2 r u_{n}^{2} t} I_{0}\left(2 u_{n}^{2} t\right)^{r}-1+e^{-u_{n}^{2} t}\right) \frac{d t}{t} \\
& =\int_{1}^{\infty}\left(\theta_{\Lambda_{n}}\left(u_{n}^{2} t\right)-1\right) \frac{d t}{t}-\int_{1}^{\infty} u_{n}^{r} e^{-2 r u_{n}^{2} t} I_{0}\left(2 u_{n}^{2} t\right)^{r} \frac{d t}{t}+\int_{1}^{\infty} e^{-u_{n}^{2} t} \frac{d t}{t} .
\end{aligned}
$$

In the last row, the third integral clearly goes to zero as $n \rightarrow \infty$. For the first integral in the same row we have

$$
\int_{1}^{\infty}\left(\theta_{\Lambda_{n}}\left(u_{n}^{2} t\right)-1\right) \frac{d t}{t} \rightarrow \int_{1}^{\infty}\left(\theta_{A}(t)-1\right) \frac{d t}{t}
$$

in view of the pointwise convergence from Proposition 9 and the uniform integrable upper bound from Lemma 10.

The middle integral

$$
\int_{1}^{\infty} u_{n}^{r} e^{-2 r u_{n}^{2} t} I_{0}\left(2 u_{n}^{2} t\right)^{r} \frac{d t}{t}
$$

converges to

$$
\int_{1}^{\infty}(4 \pi t)^{-r / 2} \frac{d t}{t}=\frac{2}{r}(4 \pi)^{-r / 2}
$$

in view of the heat kernel convergence from Proposition 7 and Proposition 8, so then we may appeal to the Lebesgue dominated convergence theorem.

Next we show:

Proposition 12 With the notation as above and $u_{n}:=\operatorname{det}\left(\Lambda_{n}\right)^{1 / r}$, we have that

$$
\int_{0}^{1}\left(\theta_{\Lambda_{n}}\left(u_{n}^{2} t\right)-u_{n}^{r} e^{-2 r u_{n}^{2} t} I_{0}\left(2 u_{n}^{2} t\right)^{r}\right) \frac{d t}{t} \rightarrow \int_{0}^{1}\left(\theta_{A}(t)-(4 \pi t)^{-r / 2}\right) \frac{d t}{t}
$$

as $n \rightarrow \infty$. 
Proof. For fixed $t$ we have the pointwise convergence as $n \rightarrow \infty$

$$
\theta_{\Lambda_{n}}\left(u_{n}^{2} t\right)-u_{n}^{r} e^{-2 r u_{n}^{2} t} I_{0}\left(2 u_{n}^{2} t\right)^{r} \rightarrow \theta_{A}(t)-(4 \pi t)^{-r / 2} .
$$

It remains therefore to exhibit uniform (for $n>>1$ ) integrable bounds on the integrands. This can be done in the same way as in the proof of Proposition 9. In order to make the bound obtained there, in terms of $\lambda$, integrable for $0 \leq t \leq 1$, we just need to choose $n_{0}$ large so that $n_{0} a / 4>r / 2$.

Finally we recall from [CJK10]:

Proposition 13 For $u \in \mathbb{R}$ we have the asymptotic formula

$$
\int_{0}^{1}\left(e^{-u^{2} t}-1\right) \frac{d t}{t}=\Gamma^{\prime}(1)-2 \log (u)+o(1)
$$

as $u \rightarrow \infty$.

We now turn to the proof of our main result, Theorem 1.

In view of Theorem [6 we have

$\log \operatorname{det} \Delta_{D T\left(\Lambda_{n}\right)}=\operatorname{det} \Lambda_{n} \cdot c_{r}-\int_{0}^{\infty}\left(\theta_{\Lambda_{n}}(t)-\operatorname{det} \Lambda_{n} \cdot e^{-2 r t} I_{0}(2 t)^{r}-1+e^{-t}\right) \frac{d t}{t}$.

After the change of variables $t \rightarrow u_{n}^{2} t$, the second term becomes

$$
\begin{aligned}
& -\int_{0}^{\infty}\left(\theta_{\Lambda_{n}}\left(u_{n}^{2} t\right)-\operatorname{det} \Lambda_{n} \cdot e^{-2 r u_{n}^{2} t} I_{0}\left(2 u_{n}^{2} t\right)^{r}-1+e^{-u_{n}^{2} t}\right) \frac{d t}{t} \\
& =-\left[\int_{0}^{1}+\int_{1}^{\infty}\right]\left(\theta_{\Lambda_{n}}\left(u_{n}^{2} t\right)-\operatorname{det} \Lambda_{n} \cdot e^{-2 r u_{n}^{2} t} I_{0}\left(2 u_{n}^{2} t\right)^{r}-1+e^{-u_{n}^{2} t}\right) \frac{d t}{t} .
\end{aligned}
$$

In view of Propositions 11, 12, and 13, this integral equals

$$
\begin{aligned}
& -\int_{1}^{\infty}\left(\theta_{A}(t)-1\right) \frac{d t}{t}+\frac{2}{r}(4 \pi)^{-r / 2}-\int_{0}^{1}\left(\theta_{A}(t)-(4 \pi t)^{-r / 2}\right) \frac{d t}{t} \\
& -\Gamma^{\prime}(1)+2 \log \left(u_{n}\right)+o(1) .
\end{aligned}
$$

Keeping in mind that $u_{n}=\left(\operatorname{det} \Lambda_{n}\right)^{1 / r}$ and identifying the constant terms appearing in the meromorphic continuation of $-\zeta^{\prime}(0)=\log \operatorname{det} \Delta_{A \mathbb{Z}^{r} \backslash \mathbb{R}^{r}}$, the main theorem is proved; cf. equation (15) of [CJK10] with $V(A)=\operatorname{det} A=1$. 


\section{$5 \quad$ Proof of the corollaries}

To prove the corollaries in the introduction we recall the statements from the literature that we use.

For Corollary 2 note that the height has a global minimum for the hexagonal lattice $A_{2}$ in dimension 2 as is well-known and for the f.c.c. lattice $A_{3} \cong D_{3}$ in dimension 3 by the rigorous numerics of Sarnak-Strömbergsson in [SS06]. Hence for any unimodular lattice $L$ in the respective dimensions

$$
\begin{aligned}
& h(L) \geq h\left(A_{2}\right), \text { so } \log \operatorname{det} \Delta_{L} \leq \log \operatorname{det} \Delta_{A_{2}} \\
& h(L) \geq h\left(D_{3}\right), \text { so } \log \operatorname{det} \Delta_{L} \leq \log \operatorname{det} \Delta_{D_{3}} .
\end{aligned}
$$

As already remarked the two leading terms in the asymptotics in Theorem 1 are shape-independent, and so Corollary 2 follows from these remarks arguing with convergent subsequences in view of the compactness.

For Corollary 3 note that Corollary 1 on p. 119 in [SS06] implies that

$$
\log \operatorname{det} \Delta_{M}<\gamma-\log 4 \pi+\frac{2}{r}<-0.95
$$

where $M$ is an $r$-dimensional flat torus of volume 1 and $\gamma$ is Euler's constant $\gamma \approx 0.577$. In view of this statement, let us replace $\Lambda_{n}$ by a convergent subsequence. Then by Theorem 1 and the matrix-tree theorem, we have

$$
\tau\left(D T\left(\Lambda_{n}\right)\right)=\frac{\operatorname{det}^{\prime} \Delta_{D T\left(\Lambda_{n}\right)}}{\operatorname{det} \Lambda_{n}} \leq \frac{\left(\operatorname{det} \Lambda_{n}\right)^{2 / r-1}}{4 \pi} \exp \left(\operatorname{det} \Lambda_{n} \cdot c_{r}+\gamma+2 / r\right)
$$

for all sufficiently large $n$. This concludes the proof of Corollary 3 ,

Finally, it may be of interest to mention another estimate in [SS06]:

$$
h(L) \geq 4 \sqrt{\frac{\pi}{r}}\left(\frac{\sqrt{r / 2 \pi e}}{m(L)}\right)^{r}(1+o(1))
$$

where $m(L)$ is the length of the shortest non-zero vector in the lattice $L$. Recall that being the densest regular packing is equivalent to being the lattice with co-volume 1 which maximizes the length of the shortest nonzero vector.

\section{References}

[CJK10] Chinta, G., Jorgenson, J., Karlsson, A., Zeta functions, heat kernels and spectral asymptotics on degenerating families of discrete tori, Nagoya Math. J. 198 (2010), 121-172 
[Ch97] Chiu, P.: Height of flat tori. Proc. Am. Math. Soc. 125, 723-730 (1997)

[CY99] Chung, F., and Yau, S.-T., Coverings, heat kernels and spanning trees, Electronic J. Combin. 6 (1999) Research Paper 12, 21 pp.

[CS99] Conway, J.H., Sloane, N.J.A.: Sphere Packings, Lattices and Groups, (3rd edn) Grundl. Math. Wiss. 290, New York: Springer Verlag 1999

[DM06] Dodziuk, J., Mathai, V., Kato's inequality and asymptotic spectral properties for discrete magnetic Laplacians, In: The ubiquitous heat kernel, Contemporary Math. AMS, 398, (2006), 269-297.

[DD88] Duplantier, B., David, F., Exact partition functions and correlation functions of multiple Hamiltonian walks on the Manhattan lattice, Journal of Statistical Physics, 51 (1988), 327-434.

[L] Lück, Wolfgang, $L^{2}$-invariants: theory and applications to geometry and $K$-theory. Ergebnisse der Mathematik und ihrer Grenzgebiete. 3. Folge. A Series of Modern Surveys in Mathematics, 44. SpringerVerlag, Berlin, 2002. xvi+595 pp. ISBN: 3-540-43566-2

[M] McKay, Brendan D., Spanning trees in regular graphs. European J. Combin. 4 (1983), no. 2, 149-160.

[OPS88] Osgood, B., Phillips, R., Sarnak, P.:Extremals of determinants of Laplacians. J. Funct. Anal. 80, 148-211 (1988)

[S90] Sarnak, P.:Determinants of Laplacians; Heights and Finiteness. In: Analysis, et cetera, pp. 601-622, ed. by P.H. Rabinowitz, E. Zehnder. Boston, MA: Academic Press 1990

[SS06] Sarnak, P. and Strömbergsson, A., Minima of Epstein's Zeta function and heights of flat tori, Invent. math. 165, 115-151 (2006)

Gautam Chinta

Department of Mathematics

The City College of New York

Convent Avenue at 138th Street

New York, NY 10031

U.S.A.

e-mail: chinta@sci.ccny.cuny.edu 
Jay Jorgenson

Department of Mathematics

The City College of New York

Convent Avenue at 138th Street

New York, NY 10031

U.S.A.

e-mail: jjorgenson@mindspring.com

Anders Karlsson

Section de mathématiques

Université de Genève

2-4 rue du Lièvre

1211 Genève 4

Switzerland

e-mail: Anders.Karlsson@unige.ch 